\title{
Occurrence Distribution of Polar Cap Patches: Dependences on UT, Season and Hemisphere
}

\section{AUTHOR(S):}

Kagawa, A.; Hosokawa, K.; Ogawa, Y.; Ebihara, Y.; Kadokura, A.

\section{CITATION:}

Kagawa, A.... [et al]. Occurrence Distribution of Polar Cap Patches: Dependences on UT, Season and Hemisphere. Journal of Geophysical Research: Space Physics 2021, 126(1): e2020JA028538.

\section{ISSUE DATE:}

2021-01

URL:

http://hdl.handle.net/2433/267746

\section{RIGHT:}

(c) 2020. American Geophysical Union. All Rights Reserved.; The full-text file will be made open to the public on 13 July 2021 in accordance with publisher's 'Terms and Conditions for Self-Archiving'. 


\section{JGR Space Physics}

\section{RESEARCH ARTICLE \\ 10.1029/2020JA028538 \\ Key Points: \\ - Statistics show that the occurrence rate of patches is a complex function of UT, season and hemisphere \\ - But, the occurrence distribution can be interpreted by the spatial overlap between the high-latitude convection and the solar terminator \\ - Outstanding interhemispheric difference is found in the UT and seasonal distribution of patches}

Correspondence to:

K. Hosokawa,

keisuke.hosokawa@uec.ac.jp

Citation:

Kagawa, A., Hosokawa, K., Ogawa, Y., Ebihara, Y., \& Kadokura, A. (2021). Occurrence distribution of polar cap patches: dependences on UT, season and hemisphere. Journal of Geophysical Research: Space Physics, 126, e2020JA028538. https://doi. org/10.1029/2020JA028538

Received 31 JUL 2020 Accepted 20 NOV 2020
(C) 2020. American Geophysical Union. All Rights Reserved.

\section{Occurrence Distribution of Polar Cap Patches: Dependences on UT, Season and Hemisphere}

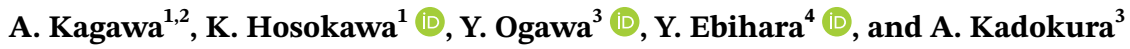 \\ ${ }^{1}$ University of Electro-Communications, Chofu, Japan, ${ }^{2}$ Now at Nippon Hoso Kyokai (NHK), Shibuya, Japan, ${ }^{3}$ National \\ Institute of Polar Research, Tachikawa, Japan, ${ }^{4}$ RISH, Kyoto University, Uji, Japan
}

\begin{abstract}
Polar cap patches are islands of enhanced electron density in the polar cap F region ionosphere, which sometimes affect the propagation of trans-ionospheric radio waves. Considering the intake of daytime sunlit plasma by the high-latitude convection as the primary cause of patches, the spatial overlap between the convection and the daytime sunlit plasma should be one of the critical factors controlling the generation of patches. To confirm this hypothesis, we statistically investigated the UT and seasonal distributions of patch occurrence frequency in both the hemispheres by using in situ plasma density data from the Swarm satellite. As a result, it was found that the occurrence distribution of patches is a complex function of UT, season and hemisphere, but it can be mostly interpreted by the spatial overlap between the high-latitude convection and the solar terminator. This suggests that polar cap patches are not necessarily phenomena that occur only during winter months. That is, patches can often be observed even in periods away from the winter solstice if the location of solar terminator in the magnetic coordinate system is appropriate for the generation of patches. For example, in the southern hemisphere, where the offset between the geographic and magnetic poles is larger than that in the northern hemisphere, the highest patch occurrence rate is obtained around the equinoctial periods. These results indicate that it is needed to take these dependences into account when we discuss and predict the space weather impacts of patches on the trans-ionospheric radio propagation.
\end{abstract}

Plain Language Summary In the upper part of the ionosphere at the highest latitudes of the Earth, we often observe isolated chunk of high-density plasma at around $300 \mathrm{~km}$ altitude. Those localized regions of enhanced plasma density is called "polar cap patches". Polar cap patches are known to occur when the interplanetary magnetic field (IMF) is directed southward and the magnetosphere-ionosphere coupling system becomes open to the energy input from the solar wind. Of course, the direction of IMF is an important factor which determines the production of patches. But, we still do not know what controls the diurnal and seasonal variations of patch activity. To answer this question, we have carried out a statistical analysis of the occurrence probability of polar cap patches by using the in situ plasma density data from the low-Earth orbiting satellite Swarm. The statistics clearly show that the diurnal and seasonal variations of patch activity are strongly characterized by the spatial overlap between the high-latitude convection and the high density source plasma in the sunlit region. The statistics also demonstrate the existence of remarkable interhemispheric difference in the patch occurrence distribution, which can be interpreted by the difference in the offset between the geographic and geomagnetic poles between the two hemispheres.

\section{Introduction}

\subsection{Polar Cap Patches}

Polar cap patches are known as regions in the polar cap F region ionosphere where the electron density locally increases at least twice as much as the background value in the surrounding area (Crowley, 1996 and references therein). Patches have so far been observed using ground-based observation techniques such as ionosondes (Weber et al., 1984), incoherent scatter radars (Pedersen et al., 2000), coherent scatter radars (Milan et al., 2002), all-sky airglow imagers (Hosokawa et al., 2019 and references therein), and GPS-TEC observations (Zhang et al., 2013). In recent years, in situ plasma density measurements onboard low-altitude satellites have also been insensitively used to investigate the statistical characteristics of polar cap patches. (e.g., Chartier et al., 2018; Noja et al., 2013; Spicher et al., 2017). 


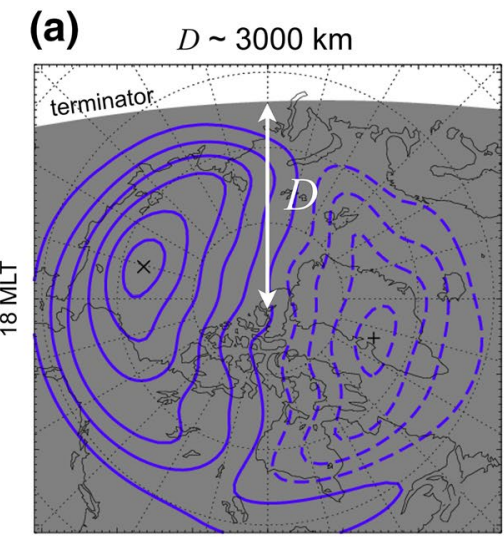

24 MLT

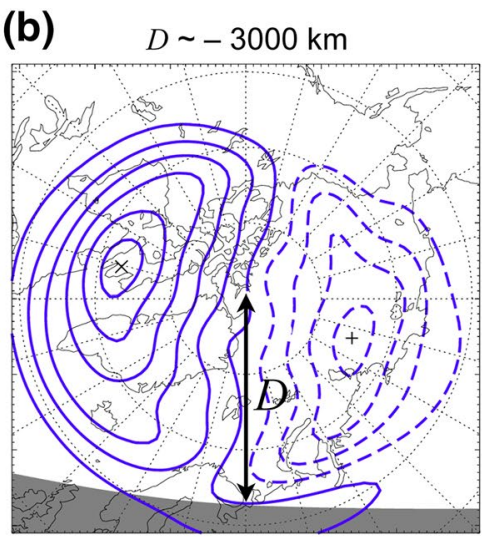

24 MLT

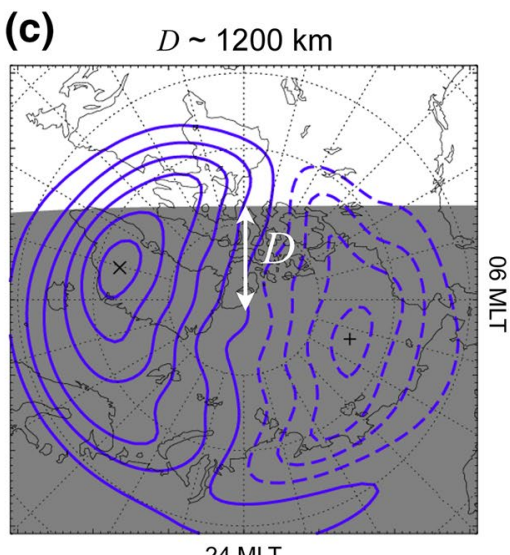

$24 \mathrm{MLT}$

Figure 1. Schematic illustration for the relationship between the high-latitude convection pattern and the location of solar terminator for three different conditions. Typical twin-cell potential contours derived from the SuperDARN map potential data are used.

Previous studies suggested that the dense plasma in the dayside sunlit area is the primary source of patches. Those plasmas are captured as individual patches near the dayside cusp by the twin-cell plasma convection, especially its antisunward flowing part in the polar cap, established when the interplanetary magnetic field (IMF) is directed southward (Carlson, 2012 and references therein). At the same time, however, it has been reported that ionization due to soft electron precipitation associated with transient aurora in the cusp such as Poleward Moving Aurora Forms (PMAFs: Fasel, 1995) can generate relatively low-density patches (Hosokawa et al., 2016).

It has also been well-known that there exist small-scale plasma density irregularities within patches (e.g., Hosokawa et al., 2009), and these irregularities may affect global navigation satellite systems (GNSS) such as GPS (Clausen et al., 2016; Jin et al., 2014; Moen et al., 2013). Hence, revealing the factors that control the occurrence (i.e., production) of patches is necessary for understanding/predicting the space weather impact in the polar cap ionosphere. In particular, clarifying the seasonal and UT dependencies of the patch occurrence frequency will allow us to expound on the discussion about the main generation mechanism of patches. In this study, we aim at identifying the factors characterizing the occurrence distribution of patches by statistically analyzing in situ electron density observations from a low-Earth orbit satellite.

\subsection{Favorable Condition for Patch Generation}

Before showing the main statistical results using the satellite data, let us review the factors that can control the seasonal and UT variations in the patch occurrence frequency. Considering the intake of daytime sunlit plasma by the high-latitude convection as the primary cause of the patch generation, the spatial (positional) relationship between the twin-cell convection pattern, especially its antisunward component, and the terminator is a critical factor for the patch production process. This is simply because if there is no overlap between the daytime sunlit plasma (located on the sunward side of the solar terminator) and the antisunward convection, intake of dense plasma never happens; thus, polar cap patches are not allowed to appear. Figure 1 schematically shows the positional relationship between the twin-cell convection pattern and the solar terminator at the ground level. The white (gray) part shows the sunlit (dark) hemisphere and the boundary between these two regions is the solar terminator on the ground. Further, this schematic diagram shows contours of typical high-latitude twin-cell convection pattern during southward IMF conditions, obtained by expanding the data from the SuperDARN radars (Chisham et al., 2007) using a spherical-harmonics fitting (Ruohoniemi \& Baker, 1998).

Figure 1a shows a case when the entire polar cap is in the dark hemisphere, as seen during winter solstice periods. In this case, we cannot expect patches to be generated because the high-latitude convection cannot reach the source plasma distributed on the sunward side of the terminator. In contrast, Figure $1 \mathrm{~b}$ shows a condition in which the entire polar cap is in the sunlit hemisphere, as seen during summer solstice periods. In this situation, the high-density plasma is distributed throughout the entire polar cap; thus, it is difficult 

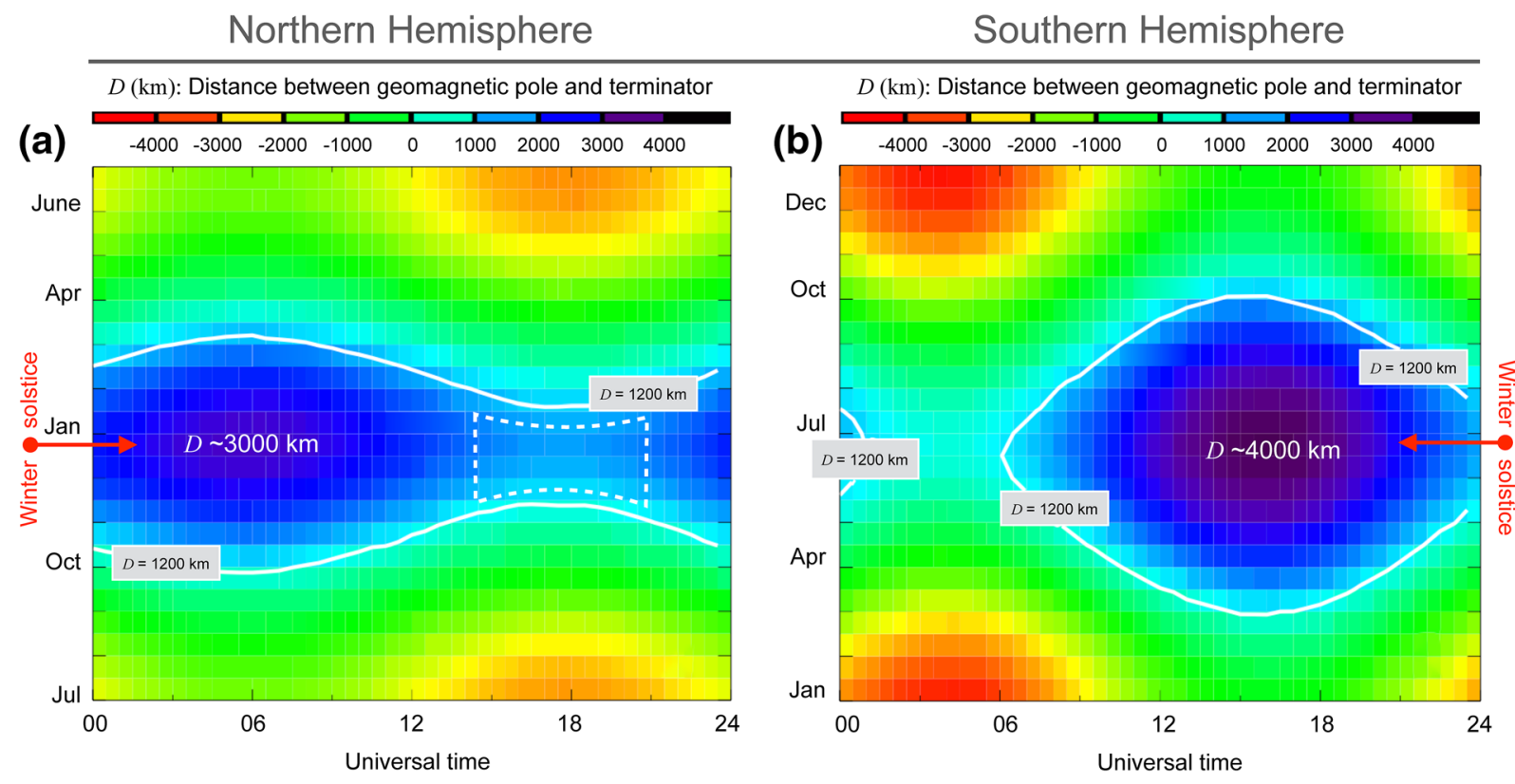

Figure 2. Distribution of $D$ as a function of UT and season in the northern hemisphere (a) and the southern hemisphere (b).

to distinguish localized electron density increase of patches from the elevated background. In other words, to generate patches, there should be a spatial overlap between the source plasma in the sunlit area and the high-latitude convection. In addition, the central polar cap should not be illuminated by the Sun. The situation shown in Figure 1c satisfies these requirements. In this case, the solar terminator is located close to the dayside cusp, which is a favorable condition for the production of patches.

Following the discussion in the previous part, we could introduce a proxy value for representing the expected occurrence rate of patches. Here, we propose to quantify the extent of spatial overlap between the source plasma and the high-latitude convection by using a distance $D$ shown in Figure 1. $D$ is defined as a distance between the geomagnetic pole, that is the center of plasma convection (in this case, the Altitude Adjusted Corrected GeoMagnetic [AACGM] magnetic pole, Baker \& Wing, 1989), and the solar terminator on the ground. Note that we assume that $D$ is positive (negative) when the terminator is in the sunward (antisunward) side of the AACGM geomagnetic pole. In Figure 1c, which presents a favorable situation for patch generation, $D$ is about $1,200 \mathrm{~km}$. This value of $D=1,200 \mathrm{~km}$ corresponds to the distance from the AACGM pole to the cusp. That is, patches are produced most efficiently when the demarcation line between the sunlit and dark hemispheres (i.e., terminator) is close to the cusp (i.e., throat of the antisunward convection). In this study, we discuss the occurrence distribution of patches by considering how the value of $D$ depends on season, UT and hemisphere.

\subsection{Possible Interhemispheric Asymmetry in Patch Occurrence}

Figure 2 shows how the distance $D$ changes depending on season and UT in the northern (Figure 2a) and southern (Figure $2 \mathrm{~b}$ ) hemispheres. Note that the center in the vertical axis corresponds to the local winter solstice. The contours of $D=1,200 \mathrm{~km}$, which represents the favorable situation for patch generation, are shown with the white lines. That is, patches are expected to be generated in the periods around these white lines (i.e., light blueish areas). Such favorable periods are present generally in winter in both the hemispheres, but, in some UT intervals, the condition is not satisfied even during the winter solstice period. This bias is particularly pronounced in the southern hemisphere. This is directly attributable to the larger offset between the geographic and AACGM magnetic poles (referred to as the "offset" hereafter) in the southern hemisphere. As of 2015 , the offset was about $7^{\circ}$ in the northern hemisphere and $16^{\circ}$ in the southern hemisphere (Baker \& Wing, 1989). Because of this interhemispheric difference in the offset, $D$ in the southern hemisphere is more variable with season than in the northern hemisphere. The diurnal variation (i.e., UT 
variation) of $D$ is also larger in the southern hemisphere. These results demonstrate that the variability of the spatial overlap between the source plasma in the sunlit area and the high-latitude convection cannot be ignored in discussing the seasonal and UT variations of patch occurrence rate. It is also suggested that the difference in the "offset" between the two hemispheres would introduce an interhemispheric asymmetry in the occurrence distribution of patches.

In the past literature, Coley and Heelis (1998) predicted that patches tend to occur more frequently when the distance between the cusp and the solar terminator is closer. Later, Noja et al. (2013) suggested that the difference in the offset may be the reason for more frequent appearance of patches in the southern hemisphere. Coley and Heelis (1998) derived the seasonal and UT dependences of patches in both the hemispheres. However, the amount of satellite data used was only about 19 months, then the statistical significance was insufficient. Although these previous studies implied an importance for considering the offset when discussing the patch occurrence distribution, there was no quantitative statistical analysis of the seasonal and UT variation of patches demonstrating the causal relationship with the offset. Later, David et al. (2016) performed a statistical analysis using the GPS-TEC database showing the role played by the offset in characterizing the seasonal and UT variation of patches. More recently, David et al. (2019) carried out similar statistics using the GPS-TEC data in both the hemispheres and clearly demonstrated the existence of predicted interhemispheric asymmetry in the density of patches. They implied that the relationship between the solar terminator and the dayside convection turn-around point is the primary key factor for fully understanding the climatology of patches seen in the two hemispheres. In their study, it was also explicitly pointed out that their results are distinctly different from those of other studies of patches based on in situ satellite measurements of electron density, such as Noja et al. (2013) and Chartier et al. (2018). Thus, it is still needed to clarify the existence of similar interhemispheric difference in the climatology of patches seen in the in situ satellite data, which will contribute to the further understanding of the generation mechanism of patches.

In this study, we use in situ plasma density data from the ESA's Swarm satellites for four years to derive the seasonal and UT variations of patch occurrence probability in a statistical fashion. In particular, we reveal whether the seasonal and UT distribution of patch occurrence frequency exhibit the predicted interhemispheric asymmetry. We also confirm the existence of this interhemispheric asymmetry by carrying out an additional statistics of patches using ground-based airglow observations in Antarctica.

\section{Materials and Methods}

In this study, we perform a statistical analysis of the patch occurrence distribution using the plasma density data obtained by the Langmuir Probe (Buchert et al., 2015) onboard the Swarm satellites (Friis-Christensen et al., 2008). The Swarm mission operates three satellites equipped with same instruments (A, B, and C) flying in formation at the low-Earth polar orbit. The inclination of the orbits is respectively $87.4^{\circ}$ for Swarm $\mathrm{A}$ and $\mathrm{C}$ and $88.0^{\circ}$ for Swarm B, which well covers the polar cap latitudes. The average orbital altitude is $\sim 460 \mathrm{~km}$ for Swarm A and C and $\sim 510 \mathrm{~km}$ for Swarm B, respectively. These altitudes of observations correspond to the topside of the $\mathrm{F}$ region where the density variation associated with patches can still be observed. In this study, we make use of the in situ plasma density data obtained by Swarm A with a time resolution of $2 \mathrm{~Hz}$ for the statistical analyses. To pick up patches from the plasma density data from the Langmuir probes onboard Swarm A, we followed the procedure proposed by Spicher et al. (2017). Spicher et al. (2017) developed a sophisticated automated patch detection algorithm named Polar Cap Patch Detection Algorithm (PCPDA). PCPDA extracts patches when the local plasma density is two times larger than the background level. This approach is not so different from similar patch extraction techniques, but PCPDA introduces a new method for obtaining the background level so that patches can be detected even if the background electron density is not sufficiently small. This method was strictly tested by Spicher et al. (2017) and it was confirmed that the statistical characteristics of patches picked up through PCPDA are well consistent with those in the previous literatures of patches.

The primary target of our statistical analysis is the high-density patch whose source is sunlit dense plasma on the dayside. Therefore, relatively lower density patches, for example the ones produced through impact ionization by the auroral electron precipitations, should be excluded from the statistical analysis. However, 


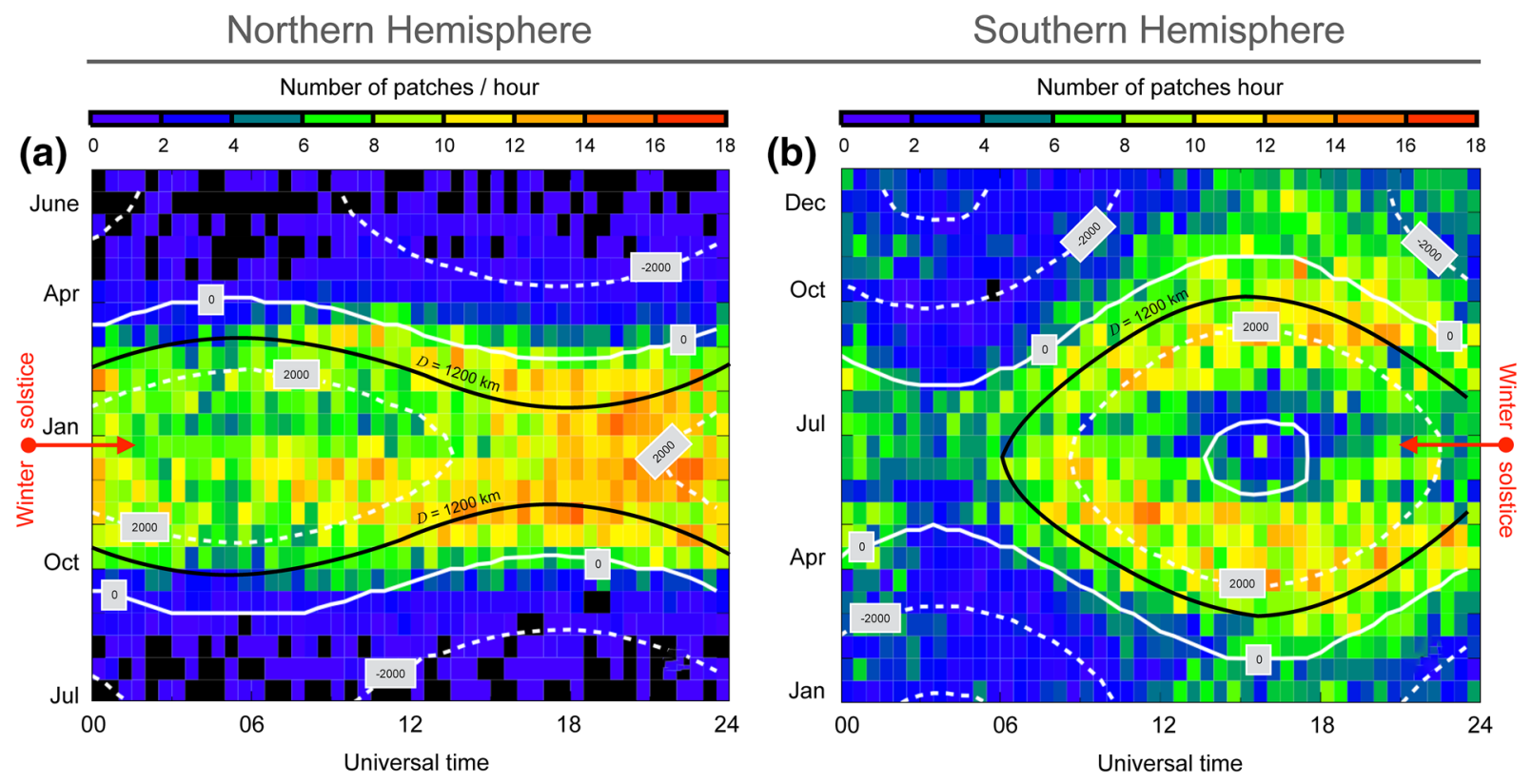

Figure 3. Statistical distribution of the number of patches detected by the Swarm A satellite in the northern hemisphere (a) and the southern hemisphere (b). Contours of several specific $D$ values are superimposed for comparison purpose.

the original PCPDA often picks up patches whose density is much smaller than those of the sunlit plasma. This is simply because there are times when the background density is low due to the effect of the offset between the geographic and AACGM poles. During such periods, lower-density patches can be extracted as peaks twice larger than the small background level. This problem was pointed out by Chartier et al. (2018). In order to overcome this problem and to correctly extract patches of dayside origin, we employed an additional criteria in the plasma density of $5.0 \times 10^{4} \mathrm{~cm}^{-3}$ for the final selection of patches. This value was determined based on the plasma density near the terminater at an altitude $\sim 460 \mathrm{~km}$, which is the orbital altitude of Swarm A. Namely, we have checked all the candidate patches extracted by PCPDA and remove ones whose plasma density is lower than $5.0 \times 10^{4} \mathrm{~cm}^{-3}$ from the final list for statistics. The densities of patches extracted by the current procedure were larger than $1.0 \times 10^{5} \mathrm{~cm}^{-3}$ in most cases, which is roughly twice the additionally employed lower limit. The maximum density of the extracted patches was as large as $2.5 \times 10^{5} \mathrm{~cm}^{-3}$. These values are sufficiently larger than the lower limit of $5.0 \times 10^{4} \mathrm{~cm}^{-3}$; thus, the statistics mainly covers dense patches of dayside origin. Still, however, we need to keep in mind that we may missed some low-density patches seen on the nightside whose density had already decreased below the threshold due to the long-distance travel from the dayside sunlit area.

To validate the statistic using the Swarm data, we have carried out another statistics using data from optical observations with an all-sky airglow imager in the southern hemisphere. We made use of a low-cost Watec Monochromatic Imager (WMI: Ogawa et al., 2020) which has been operative at McMurdo Station $\left(77.5^{\circ} \mathrm{S}\right.$, $166.4^{\circ}$ E, AACGM latitude $-79.9^{\circ}$ ) in Antarctica. One of the WMI imagers at McMurdo captures an airglow emission from oxygen atom at $630.0 \mathrm{~nm}$ which is known to be proportional to the electron density in the $\mathrm{F}$ region. The imager is operative during winter months from April to September, and the observation is continuous for $24 \mathrm{~h}$ during the period near the winter solstice.

\section{Results}

Using the in situ plasma density data from Swarm A over a 4-year period from January 2014 to December 2017, we performed a statistical analysis of the occurrence distribution of polar cap patches in both the hemispheres. For the statistics, we only picked up patches observed in the region where the magnitude of the AACGM latitude is larger than $70^{\circ}$. Figure 3 shows the seasonal and UT distribution of the occurrence of patches in the northern (Figure 3a) and southern (Figure 3b) hemispheres. The vertical axis is day of year 

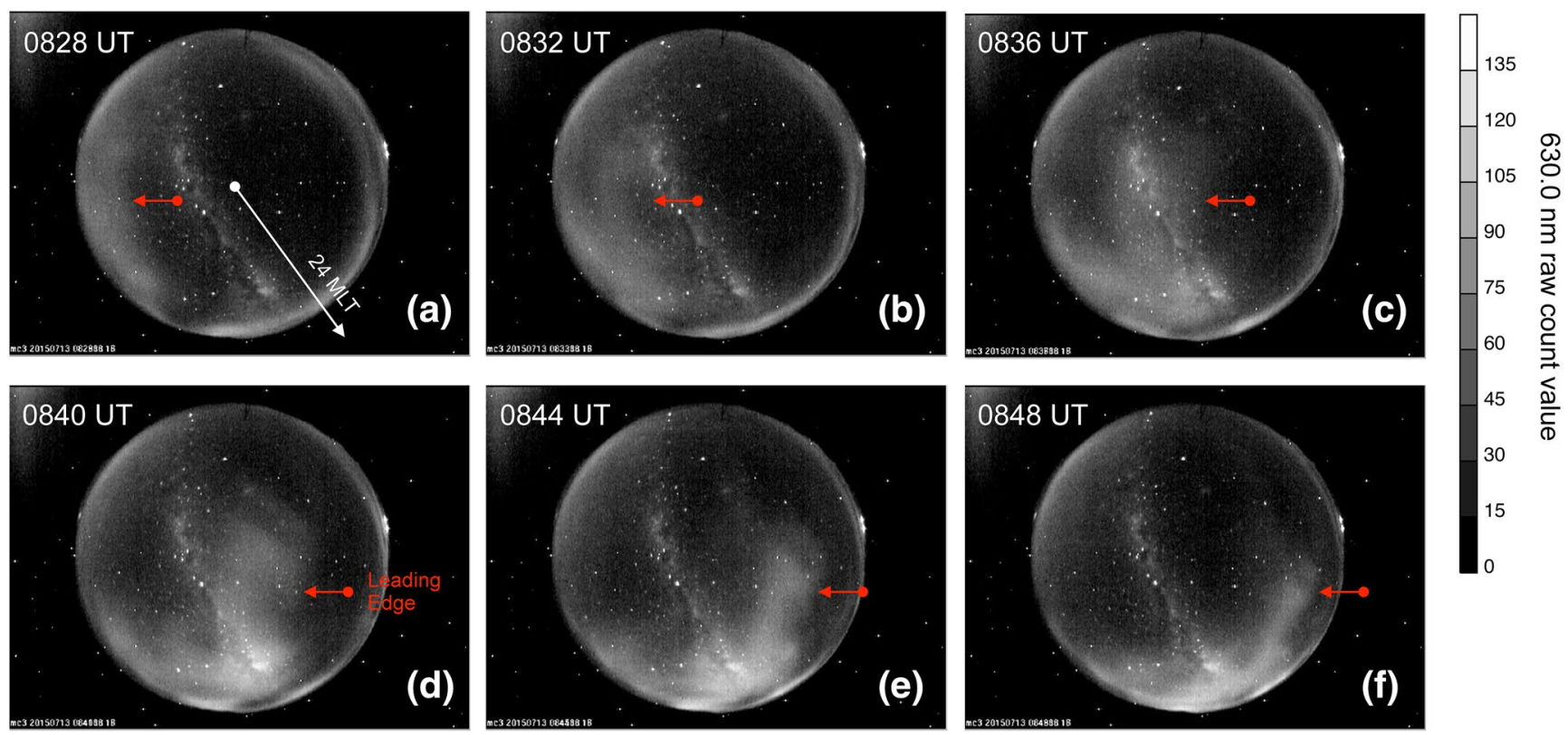

Figure 4. Sequence of $630.0 \mathrm{~nm}$ all-sky airglow images from the WMI imager at McMurdo Station in Antarctica during 20 min from 0828 to 0848 UT on July 13, 2015. The red arrow indicated the leading edge of a polar cap airglow patch passing through the field-of-view. WMI, Watec Monochromatic Imager.

in both the panels, where the center of the axis is the local winter solstice of each hemisphere (i.e., December in the northern and June in the southern hemisphere, respectively). The occurrence rate is derived in each bin, whose size is $30 \mathrm{~min}$ in horizontal (UT) $\times 15$ days in vertical axes, as the total number of patches extracted by PCPDA divided by the total hours of observations. Note that since we have used 4 years of Swarm observations, the data cover all the areas of polar cap almost equally where the average observation time for each statistical bin is a few hours on average and at least longer than $2 \mathrm{~h}$ for all the bins. To further investigate the relationship between the patch occurrence frequency and $D$, we overplotted the contours of $D$ shown in Figure 2 every 2,000 km as white lines. In addition, we overlaid the contour of $D=1,200 \mathrm{~km}$ with the thick solid line.

Figure 3a demonstrates that the occurrence of patches in the northern hemisphere is concentrated in a half a year period from October to March and there is no noticeable patch activity in summer. It is also interesting to note that, although patches are generally more active in winter, their occurrence significantly varies with UT. Near the winter solstice, for example, the occurrence is higher in the later UT interval from 1500 to 2400 UT and is significantly reduced (only $30 \%-50 \%$ of the maximum) in the earlier UT period from 0300 to 1200 UT. Namely, the occurrence distribution of patches is a complex function of season and UT. In the southern hemisphere, such dependences on season and UT are more obvious than in the northern hemisphere. Figure 3b shows that southern hemispheric patches are observed in a wide range of seasons, with a particularly higher probability in the equinoctial periods (September, October, March, and April). Even in those periods, however, the occurrence rate is not always high throughout the entire UT interval; there is almost no patch activity in the earlier UT interval (from 0300 to $0800 \mathrm{UT}$ ). During periods near the winter solstice, the UT dependence of patch activity is more complicated; there is a semi-diurnal variation in the occurrence. In particular, there is a clear minimum in the occurrence of patches from 1200 to 1800 UT, which is called "hole" hereafter (this term was first introduced by David et al., 2019). In summary, southern hemispheric patches can be detected over a longer period of time than in the northern hemisphere (patches are detected even near the summer solstice) and the UT variability is much more pronounced in the southern hemisphere.

Hereafter we introduce an observation of airglow patches in the southern hemisphere and show the result of statistics using those optical measurements. Figure 4 shows $630.0 \mathrm{~nm}$ consecutive all-sky images every 


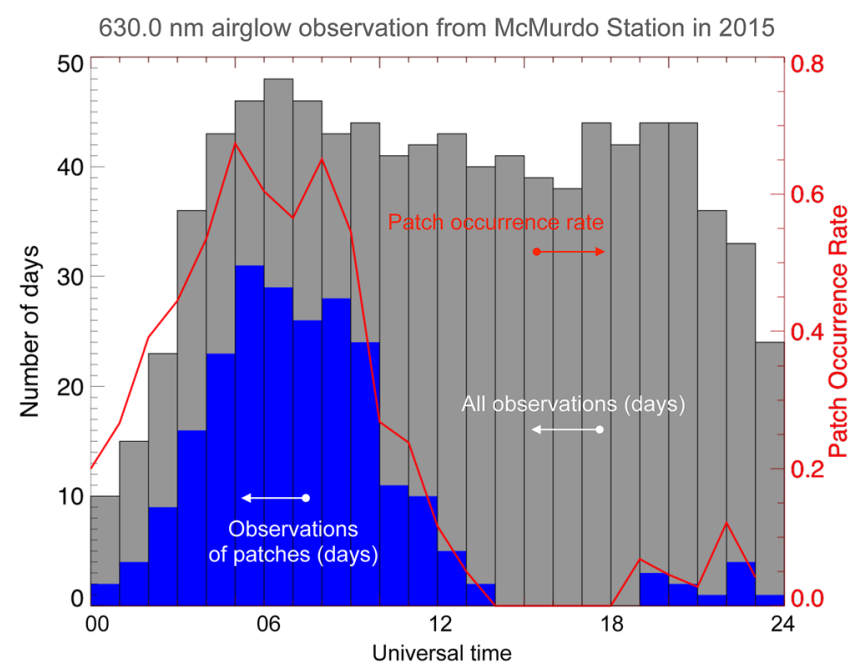

Figure 5. Occurrence distribution of $630.0 \mathrm{~nm}$ airglow patches observed by the WMI at McMurdo station during the winter period in 2015. The occurrence rate of airglow patches is shown by the red curve. WMI, Watec Monochromatic Imager.
4 min from 0828 to 0848 UT on July 13, 2015 during which an airglow patch passed through the field-of-view of the imager. The original temporal resolution (and exposure time) of the $630.0 \mathrm{~nm}$ airglow measurement is $\sim 4 \mathrm{~s}$, but we have integrated the raw images for $2 \mathrm{~min}$ to increase the signal-to-noise ratio. During this time interval, McMurdo Station was located on the night side within the polar cap. The white arrow in Figure 4a gives the direction of magnetic midnight (i.e., 24 MLT). The IMF $\mathrm{Bz}$ obtained from the OMNI database (https://omniweb.gsfc.nasa.gov) ranged from -6 to $-2 \mathrm{nT}$ in this interval, which is a favorable condition for the generation of patches. It is seen that a diffuse structure is passing across the field-of-view of the imager which is a signature of a polar cap patch in the $630.0 \mathrm{~nm}$ airglow. This airglow patch drifted roughly in the antisunward direction toward 24 MLT. Figure 5 plots the UT variation of the patch occurrence distribution around the winter solstice derived by using airglow data from 3 months centered on the winter solstice in 2015 (from May 8 to August 6). 516 airglow patches were detected by visual inspection in this 3 months interval. In order to calculate the frequency of patch occurrence, the total number of hours, in which the airglow observations of patches are possible (i.e., no contamination of sunlight and moonlight, and the good weather condition), is calculated for each UT bin (gray histogram in Figure 5). We also derived the number of days when at least one patch was detected in each UT bin (blue histogram in Figure 5). Then, the frequency of patch occurrence was derived by dividing the blue histogram by the gray histogram (red line in Figure 5).

Focusing on the occurrence rate of patches (red line in Figure 5), it can be seen that the maximum occurrence is obtained in the time period of 0500-0800 UT and the minimum value is at around 1500 UT. Especially, the occurrence rate is zero during $3 \mathrm{~h}$ from 1400 to $1700 \mathrm{UT}$. This time zone is almost identical to the time zone of the "hole" structure (1300-1800 UT) seen in the statistics using the Swarm data (Figure 3b). In addition, the peak of patch occurrence near the winter solstice is around 0800 UT in the satellite observations. We see a peak in the similar UT period in the statistics using the airglow observations. These correspondences further confirm that the characteristic (i.e., semi-diurnal) UT variation of patch occurrence inferred from the Swarm data surely exists in the southern hemisphere. In particular, the ground-based airglow observations imply that the occurrence frequency of southern hemispheric patches is extremely reduced in the afternoon UT interval near the winter solstice.

\section{Discussion}

The statistics using the in situ Swarm data showed a characteristic UT dependence of the patch occurrence in the periods around the winter solstice. Especially in the southern hemisphere, a complicated diurnal variation of the patch activity is seen during $\sim 60$ days centered at the winter solstice. One of the manifestations of such complicated UT variation is the "hole" structure in the occurrence of patches seen in Figure $3 b$. The existence of the hole was first discussed and predicted by Sojka et al. (1994) by analyzing the results of numerical simulation of patches. They implied that the hole is primarily caused by the combination of very small background density in the polar cap region and the absence of tongue of ionization formation during certain UT periods. Coley and Heelis (1998) also pointed out that, in the southern hemisphere, the active production of patches may be less frequent during 1400-1600 UT than in the surrounding time intervals, due to displacement of the cusp to a region with a smaller background plasma density. Later, David et al. $(2016,2019)$ confirmed the formation of the hole structure by analyzing the GPS-TEC database in a statistical fashion. They also demonstrated the existence of the interhemispheric asymmetry in the UT and seasonal distribution of patch activity. Although these previous studies, especially the recent studies by David and colleagues, have already showed the UT variation in the occurrence of patches in the GPS-TEC data, the current statistical analysis confirmed the existence of both the hole structure and remarkable interhemispheric difference by using the in situ plasma density data at the low altitude. We also confirmed 
the UT variation in the southern hemisphere by using the ground-based airglow observations. These results indicate that, irrespective of the observations method (and procedure of statistical analysis), the UT and seasonal distribution of patch activity shows a characteristic pattern shown in Figure 3 of David et al. (2019) and also Figure 3 of this study.

In the Introduction section, we proposed that the UT and seasonal variation of patch activity can be understood quantitatively by introducing a parameter called $D$, which is the distance from the AACGM pole to the terminator (described in Figure 1). This distance $D$ changes depending on UT and season due to the offset between the poles. We hypothesize that the offset between the poles dominates the UT and seasonal variation of patch occurrence distribution. To test this, we discuss below the relationship between the UT and seasonal variations of $D$ (Figure 2) and actual patch occurrence rate (Figure 3 ) in detail. First we compare the northern hemisphere case (Figure 2a vs. Figure 3a). Figure 3a shows that patches are frequently observed in the later UT intervals, say 12-24 UT. This period of high patch activity corresponds to a region of light bluish color outlined by the dashed line in Figure 2a, where the $D$ value is around 1,200 $\mathrm{km}$. In addition, there is a slight indication that the occurrence of patch is higher along the contours of $D=1,200 \mathrm{~km}$. These comparisons indicate that the spatial relationship between the source plasma and the high-latitude convection is one of the primary factors controlling the activity of polar cap patches. In the southern hemisphere, higher patch activity is seen again along the contour of $D=1,200 \mathrm{~km}$. This introduces relatively higher patch occurrence in the equinoctial period rather than the local winter solstice. This tendency is consistent with Figure 5 of Chartier et al. (2018) who stated that patches are more frequent in December in both hemispheres, but their statistical results actually show that patches are more frequent in equinox in both the hemispheres. Their statistical results can also be interpreted by introducing $D$ as a proxy for the efficiency of the patch generation process.

Finally, let us focus on the $D$ values near the hole structure seen around the winter solstice. The white contours overplotted on Figure $3 \mathrm{~b}$ indicates that $D$ values near the hole structure is about 3,000 $\mathrm{km}$ or more. In such a situation, as displayed in Figure 1a, the solar terminator is located on the dayside far away from the AACGM pole. This does not allow the high-latitude convection to capture the source plasma in the sunlit area, then almost no patch production is expected. In contrast to the southern hemisphere, the hole structure is less obvious in the northern hemisphere (Figure 3a). In actual, the distribution of $D$ shows that there is no time period in which $D$ is larger than 3,000 km (Figure 2a). This difference produces the prominent interhemispheric asymmetry in the patch activity, especially the existence of the hole structure. Sojka et al. (1994) predicted, by using numerical modeling, fewer occurrence of patches in the northern hemisphere during the 0800-1200 UT period during 40 days around the winter solstice. In our statistical results (Figure 3a), 5-12 patches were identified per hour in this time period, but the frequency of occurrence is lower than the surrounding area. That is, the slight decrease in the occurrence of patches during this time period corresponds to the hole structure in the northern hemisphere, although it is not as noticeable as that in the southern hemisphere.

Based on the statistics, we have mainly discussed the spatial overlap between the high-density plasma on the dayside and the high-latitude convection pattern as the primary controlling factor of patch occurrence. However, we should keep in mind that patches can often be produced by direct particle precipitation in the polar cap, for example, reported by Zhang et al. (2017) and Ma et al. (2018). We intended to remove contributions of such patches to the statistics by using an additional density threshold in the PCPDA algorithm of Spicher et al. (2017). However, the statistics might still contain relatively higher density patches produced by the particle precipitation. In addition, we have only used the $D$ value for expressing how effective the capturing of high-density plasma works around the dayside throat region. However, we should bear in mind that, during magnetic storms, the high-latitude convection pattern significantly expands to lower latitudes and, in such a case, the simple use of $D$ value is not able to describe the patch production process. We also need to stress that the simple use of $D$ value does not allow us to consider the effect of transportation of patches on their occurrence distribution. The convection speed surely affects the density of patches seen in the central polar cap. For instance, if the convection is slower, patch plasmas are sufficiently recombined due to the long-time travel from the dayside to the central polar cap. All of the above mentioned factors: (1) the effect of particle precipitation, (2) the size of high-latitude convection, and (3) the speed of convection, are superimposed on the "base" seasonal-UT distribution shown in the current statistics and the occurrence of patches should be determined in actual cases. 


\section{Conclusion}

We have carried out statistical analyses of the occurrence of polar cap patches using in situ plasma density data from the Swarm satellite together with ground-based airglow observations in Antarctica. From the statistics, we conclude that the occurrence of polar cap patches is controlled by $D$, which is a distance from the magnetic pole to the solar terminator. This distance $D$ is determined by the offset between the geographic and AACGM geomagnetic poles; thus, the seasonal and UT distributions of the patch occurrence change depending on the location of solar terminator in the magnetic coordinate system. This fact suggests that polar cap patches are not necessarily phenomena that occur only during winter months. That is, patches can often be observed even in the period away from the winter solstice if the location of solar terminator in the magnetic coordinate system is appropriate for the generation of patches. For example, in the southern hemisphere, where the offset is larger than that in the northern hemisphere, the highest patch occurrence rate is obtained around the equinoctial periods. The magnitude of IMF Bz is surely one of the parameters controlling the occurrence of polar cap patches. However, current statistical analyses demonstrated much stronger dependences of patch activity on UT, season and hemisphere, which are introduced by the offset between the geographic and geomagnetic poles. It is needed to take these dependences into account when we discuss and predict the space weather impacts of patches, for example their effects (code delay and scintillations) on trans-ionospheric GNSS signals.

\section{Data Availability Statement}

All the Langmuir probe data of Swarm used in the current studies are obtained from the above mentioned web page. The $630.0 \mathrm{~nm}$ all-sky airglow data from WMI are available at http://pc115.seg20.nipr.ac.jp/www/ opt $/ \mathrm{mcm} . \mathrm{html}$.

\section{Acknowledgments}

$\mathrm{KH}$ is supported by JSPS-Kakenhi (26302006, 17H02967). We thank the European Space Agency for supplying Swarm data through the https://swarmdiss.eo.esa.int portal.

\section{References}

Baker, K. B., \& Wing, S. (1989). A new magnetic coordinate system for conjugate studies at high latitudes. Journal of Geophysical Research, 94(A7), 9139-9143. https://doi.org/10.1029/JA094iA07p09139

Buchert, S., Zangerl, F., Sust, M., Andre, M., Eriksson, A., Wahlund, J., \& Opgenoorth, H. (2015). SWARM observations of equatorial electron densities and topside GPS track losses. Geophysical Research Letters, 42, 2088-2092. https://doi.org/10.1002/2015GL063121

Carlson, H. C. (2012). Sharpening our thinking about polar cap ionospheric patch morphology, research, and mitigation techniques. Radio Science, 47(4). https://doi.org/10.1029/2011RS004946

Chartier, A. T., Mitchell, C. N., \& Miller, E. S. (2018). Annual occurrence rates of ionospheric polar cap patches observed using Swarm. Journal of Geophysical Research: Space Physics, 123, 2327-2335. https://doi.org/10.1002/2017JA024811

Chisham, G., Lester, M., Milan, S. E., Freeman, M. P., Bristow, W. A., Grocott, A., et al. (2007). A decade of the Super Dual Auroral Radar Network (SuperDARN): Scientific achievements, new techniques and future directions. Surveys in Geophysics, 28, 33-109. https://doi. org/10.1007/s10712-007-9017-8

Clausen, L. B. N., Moen, J. I., Hosokawa, K., \& Holmes, J. M. (2016). GPS scintillations in the high latitudes during periods of dayside and nightside reconnection. Journal of Geophysical Research: Space Physics, 121, 3293-3309. https://doi.org/10.1002/2015JA022199

Coley, W. R., \& Heelis, R. A. (1998). Structure and occurrence of polar ionization patches. Journal of Geophysical Research, 103(A2), 2201-2208. https://doi.org/10.1029/97JA03345

Crowley, G. (1996). Critical review of ionospheric patches and blobs. In W. R. Stone (Ed.), Review of radio science 1993-1996 (p. 619). New York, NY: Oxford University Press.

David, M., Sojka, J. J., Schunk, R. W., \& Coster, A. J. (2016). Polar cap patches and the tongue of ionization: A survey of GPS TEC maps from 2009 to 2015. Geophysical Research Letters, 43, 2422-2428. https://doi.org/10.1002/2016GL068136

David, M., Sojka, J. J., Schunk, R. W., \& Coster, A. J. (2019). Hemispherical shifted symmetry in polar cap patch occurrence: A survey of GPS TEC maps from 2015-2018. Geophysical Research Letters, 46, 10726-10734. https://doi.org/10.1029/2019GL083952

Fasel, G. J. (1995). Dayside poleward moving auroral forms: A statistical study. Journal of Geophysical Research, 100(11), 11891-11905. https://doi.org/10.1029/95JA00854

Friis-Christensen, E., Lühr, H., Knudsen, D., \& Haagmans, R. (2008). Swarm An Earth Observation Mission investigating Geospace. Advances in Space Research, 41, 210-216. https://doi.org/10.1016/j.asr.2006.10.008

Hosokawa, K., Shiokawa, K., Otsuka, Y., Ogawa, T., St-Maurice, J.-P., Sofko, G. J., \& Andre, D. A. (2009). The relationship between polar cap patches and field-aligned irregularities as observed with an all-sky airglow imager at Resolute Bay and the PolarDARN radar at Rankin Inlet. Journal of Geophysical Research, 114, A3. https://doi.org/10.1029/2008JA013707

Hosokawa, K., Taguchi, S., \& Ogawa, Y. (2016). Periodic creation of polar cap patches from auroral transients in the cusp. Journal of Geophysical Research: Space Physics, 121, 5639-5652. https://doi.org/10.1002/2015JA022221

Hosokawa, K., Zou, Y., \& Nishimura, Y. (2019). Airglow patches in the polar cap region: a review. Space Science Reviews, 215, 53. https:// doi.org/10.1007/s11214-019-0616-8

Jin, Y., Moen, J. I., \& Miloch, W. J. (2014). GPS scintillation effects associated with polar cap patches and substorm auroral activity: direct comparison. Journal of Space Weather and Space Climate, 4(27), A23. https://doi.org/10.1051/swsc/2014019

Ma, Y.-Z., Zhang, Q.-H., Xing, Z.-Y., Heelis, R. A., Oksavik, K., \& Wang, Y. (2018). The ion/electron temperature characteristics of polar cap classical and hot patches and their influence on ion upflow. Geophysical Research Letters, 45, 8072-8080 https://doi.org/10.1029/2018GL079099 
Milan, S. E., Lester, M., \& Yeoman, T. K. (2002). HF radar polar patch formation revisited: summer and winter variations in dayside plasma structuring. Annales Geophysicae, 20, 487.

Moen, J., Oksavik, K., Alfonsi, L., Daabakk, Y., Romano, V., \& Spogli, L. (2013). Space weather challenges of the polar cap ionosphere. Journal of Space Weather and Space Climate, 3(26), A02. https://doi.org/10.1051/swsc/2013025

Noja, M., Stolle, C., Park, J., \& Lühr, H. (2013). Long-term analysis of ionospheric polar patches based on CHAMP TEC data. Radio Science, 48, 289-301. https://doi.org/10.1002/rds.20033

Ogawa, Y., Tanaka, Y., Kadokura, A., Hosokawa, K., Ebihara, Y., Motoba, T., et al. (2020). Development of low-cost multi-wavelength imager system for studies of aurora and airglows. Policy Sciences, 23, 100501. https://doi.org/10.1016/j.polar.2019.100501

Pedersen, T., Fejer, B., Doe, R., \& Weber, E. (2000). An incoherent scatter radar technique for determining two-dimensional horizontal ionization structure in polar cap F region patches. Journal of Geophysical Research, 105(10), 637.

Ruohoniemi, J. M., \& Baker, K. B. (1998). Response of high latitude convection to a sudden southward IMF turning. Geophysical Research Letters, 25, 2913.

Sojka, J. J., Bowline, M. D., \& Schunk, R. W. (1994). Patches in the polar ionosphere: UT and seasonal dependence. Journal of Geophysical Research, 99(A8), 14959-14970. https://doi.org/10.1029/93JA03327

Spicher, A., Clausen, L. B. N., Miloch, W. J., Lofstad, V., Jin, Y., \& Moen, J. I. (2017). Interhemispheric study of polar cap patch occurrence based on Swarm in situ data. Journal of Geophysical Research: Space Physics, 122, 3837-3851. https://doi.org/10.1002/2016JA023750

Weber, E. J., Buchau, J., Moore, J. G., Sharber, J. R., Livingston, R. C., Winningham, J. D., \& Reinisch, B. W. (1984). F layer ionization patches in the polar caps. Journal of Geophysical Research, 89, 1683.

Zhang, Q.-H., Zhang, B.-C., Lockwood, M., Hu, H.-Q., Moen, J., Ruohoniemi, J. M., et al. (2013). Direct observations of the evolution of polar cap ionization patches. Science, 339(6127), 1597-1600. https://doi.org/10.1126/science.1231487

Zhang, Q.-H., Ma, Y. Z., Jayachandran, P. T., Moen, J., Lockwood, M., Zhang, Y. L., et al. (2017). Polar cap hot patches: Enhanced density structures different from the classical patches in the ionosphere. Geophysical Research Letters, 44, 8159-8167. https://doi. $\operatorname{org} / 10.1002 / 2017$ GL073439 